\title{
Stabilisation faible d'équations d'évolution: la méthode du problème factice (cas d'une perturbation bornée)
}

\author{
Geoffrey O’Dowd*
}

\begin{abstract}
We consider an evolution equation with a nonlinear dissipation $B$. Weak stabilization of weak solutions of this equation is proven as soon as a similar result is known for a dissipation $B_{0}$ whose kernel contains the one of $B$. Applications of this principle are given to some hybrid systems.
\end{abstract}

\section{Introduction}

Considérons une équation d'évolution du type

$$
\frac{d U}{d t}+A U+B(U)=0
$$

sur un espace de Hilbert $H$, où $A$ est un opérateur linéaire non borné conservatif et $B$ un opérateur dissipatif défini sur $H$ pour laquelle nous nous intéresserons à l'aspect stabilité. Lorsque (1.1) possède des solutions, en un sens à préciser, il lui est associé un semi-groupe, généralement non contractant en l'absence de toute hypothèse de monotonie sur $B$. Or la contraction peut s'avérer un facteur décisif dans ce genre de situations, car elle permet d'une part par densité de se limiter à prouver la stabilité pour les solutions fortes et d'autre part d'utiliser le principe de LaSalle, au moins sous certaines hypothèses de précompacité des orbites.

Toutefois, la seule dissipativité entraîne l'existence de valeurs d'adhérence faibles à toute trajectoire auxquelles nous pouvons classiquement associer, par l'intermédiaire d'un passage à la limite sur une suite de solutions translatées, une fonction $\bar{U}(t)$ ayant notamment la propriété d'être une solution du problème conservatif

$$
\frac{d U}{d t}+A U=0
$$

dont la nullité a pour conséquence la stabilité faible des solutions de (1.1), comme dans [9] ou [8] où différentes techniques basées sur les mesures de Young ou sur des méthodes spectrales sont utilisées.

\footnotetext{
*Institut Elie Cartan, B.P. 239, 54506 Vandœuvre lès Nancy, France. e-mail: odowd@wanadoo.fr
} 
L'idée que nous nous proposons d'exploiter ici réside dans la simple observation que la solution $\bar{U}(t)$ de (1.2), sous l'hypothèse que son caractère conservatif provient de la nullité du terme dissipatif $B(\bar{U}(t))$, est une solution conservative de l'équation

$$
\frac{d U}{d t}+A U+B_{0}(U)=0
$$

dès lors que le 'noyau' de l'opérateur $B_{0}$ contient celui de $B$. Aussi, si toutes les solutions de (1.3) tendent fortement vers 0 , il en résultera que $\bar{U}(t)$ est identiquement nulle.

L'intérêt de cette démarche, baptisée méthode du problème factice, repose sur le fait que la question de la stabilité du problème initial peut être ramenée à celle d'un problème plus simple lorsqu'il est possible de choisir un opérateur $B_{0}$ possédant des qualités telles que la monotonie ou la linéarité.

Dans la deuxième section de cette note, nous présenterons une version abstraite de ce principe, après avoir effectué quelques rappels sur la notion de solution faible d'une équation d'évolution. Dans la troisième section, nous donnerons des applications à certains systèmes hybrides, permettant de traiter la question de la stabilisation des solutions faibles laissée en suspens dans [3] et [4].

\section{$2 \quad$ Résultats abstraits}

Nous commencerons par quelques rappels:

Proposition 2.1 Soit $H$ un espace de Hilbert, $A$ un opérateur linéaire non borné anti-adjoint sur $H$ et $B: H \rightarrow H$ un opérateur continu vérifiant

$$
\forall U \in H,\langle B(U), U>\geq 0 .
$$

On suppose que l'opérateur $A+B$, de domaine $D(A)$, engendre un semi-groupe $(S(t))_{t \geq 0}$ d'opérateurs continus sur $H$.

Pour tout $U_{0} \in H$, l'application $U: t \mapsto S(t)\left(U_{0}\right)$ est appelée solution faible de l'équation d'évolution

$$
\left\{\begin{array}{l}
\frac{d U}{d t}+A U+B(U)=0 \\
U(0)=U_{0} .
\end{array}\right.
$$

i. Lorsque $U_{0} \in D(A)$, cette solution est dite forte. C'est la seule application vérifiant les propriétés suivantes:

$$
\begin{gathered}
\forall t \geq 0, U(t) \in D(A) \\
\forall T>0, U \in W^{1, \infty}((0, T), H) \\
p . p . t \geq 0, \frac{d U}{d t}(t)+A U(t)+B(U(t))=0 \\
U(0)=U_{0}
\end{gathered}
$$


ii. Pour toute solution faible $t \mapsto U(t)$ de (2.2), la fonction énergie

$$
E\left[U_{0}\right]: t \mapsto \frac{1}{2}\|U(t)\|^{2}
$$

est décroissante sur $\mathbb{R}^{+}$. Par ailleurs, on a l'inégalité d'énergie

$$
\int_{0}^{+\infty}<B(U(t)), U(t)>d t<+\infty
$$

iii. On suppose de plus que

$B$ transforme les parties bornées de $H$ en parties bornées.

Alors toute solution faible $t \mapsto U(t)$ de (2.2) satisfait la formulation variationnelle

$$
\begin{gathered}
\forall T>0, U \in C([0, T], H), \\
\forall W \in D\left(A^{*}\right), t \mapsto<U(t), W>\in W^{1,1}((0, T), \mathbb{R}) \\
p . p . t \geq 0, \frac{d}{d t}<U(t), W>+<U(t), A^{*} W>+<B(U(t)), W>=0 .
\end{gathered}
$$

Démonstration. La partie i. est très classique (cf. [5]). Ensuite, par continuité du semi-groupe, toute solution faible est limite simple d'une suite de solutions fortes. La notion de fonction décroissante étant stable par passage à la limite simple, il suffit de prouver (2.7) lorsque $U(0) \in D(A)$, ce qui est immédiat puisqu'en effectuant le produit scalaire par $U(t)$ dans (2.5) et en intégrant,

$$
E\left[U_{0}\right](S)-E\left[U_{0}\right](T)=\int_{S}^{T}<B(U(t)), U(t)>d t .
$$

d'où (2.7).

Enfin, (2.8) résulte de (2.11) lorsque $U(0) \in D(A)$ et le cas général s'en déduit de la façon suivante: si $U_{0} \in H$, il existe une suite $U_{n} \in D(A)$ convergeant vers $U_{0}$. Avec des notations évidentes, on a alors, pour tout $t \geq 0, U_{n}(t) \rightarrow U(t)$ lorsque $n \rightarrow+\infty$ ainsi que $<B\left(U_{n}(t)\right), U_{n}(t)>\rightarrow<B(U(t), U(t)>$. Etant donné que

$$
\int_{0}^{+\infty}<B\left(U_{n}(t)\right), U_{n}(t)>d t \leq E\left[U_{n}\right](0)=\frac{1}{2}\left\|U_{n}\right\|^{2} \leq C,
$$

on peut conclure à l'aide du lemme de Fatou.

Enfin, (2.10) est immédiat pour les solutions fortes. Dans le cas général, on introduit comme ci-dessus une suite de solutions fortes $U_{n}($.$) , qui satisfont alors (2.10). En$ intégrant entre 0 et $t$, on obtient alors

$$
\begin{gathered}
<U_{n}(t), W>-<U_{n}(0), W>+ \\
\int_{0}^{t}<U_{n}(s), A^{*} W>d s+\int_{0}^{t}<B\left(U_{n}(s)\right), W>d s=0 .
\end{gathered}
$$

ESAIM: Proc., Vol. 8, 2000, 137-150 
On a $\left\|U_{n}(s)\right\| \leq\left\|U_{n}(0)\right\| \leq C$. On peut donc passer à la limite par convergence dominée dans la première intégrale, ainsi que dans la seconde, grâce à (2.9). On obtient alors

$$
<U(t), W>-<U(0), W>+\int_{0}^{t}<U(s), A^{*} W>d s+\int_{0}^{t}<B(U(s)), W>d s=0 .
$$

Il est clair que $t \mapsto \int_{0}^{t}<U(s), A^{*} W>d s+\int_{0}^{t}<B(U(s)), W>d s$ appartient à $W^{1,1}((0, T), \mathbb{R})$ et qu'on a alors $(2.10)$.

Remarque 2.1 L'hypothèse (2.9) est notamment satisfaite lorsque $B$ est localement lipschitzienne. Dans ce cas, l'opérateur $A+B$ engendre effectivement un semi-groupe: il suffit pour cela de prouver l'existence de solutions fortes à (2.2). Disposant de l'estimation a priori $\|U(t)\| \leq\left\|U_{0}\right\|$, on peut 'remplacer' l'opérateur $B$ par un opérateur $\bar{B}$ coïncidant avec $B$ sur la boule de centre 0 et de rayon $\left\|U_{0}\right\|$ pour résoudre (2.2). $B$ étant par hypothèse lipschitzienne sur cette boule, on peut aisément construire un opérateur $\bar{B}$ globalement lipschitzien sur $H$, pour lequel l'existence de solutions à l'équation (2.2) correspondante est classique.

Remarque 2.2 Supposons à nouveau $B$ localement lipschitzienne. Alors pour tout $U_{0} \in H$ la fonction $E\left[U_{0}\right]$ est dérivable sur $[0,+\infty[$ et

$$
\forall t \geq 0, E\left[U_{0}\right]^{\prime}(t)=-<B(U(t)), U(t)>
$$

Ceci est en effet immédiat pour les solutions fortes d'après (2.11). Pour les solutions faibles, on introduit une suite $U_{n}($.$) de solutions fortes convergeant simplement vers$ $U($.$) . Comme on l'a vu ci-dessus, tous les U_{n}(t)$, avec $t \geq 0$ et $n \in \mathbb{I}$, sont dans une même boule $\mathcal{B}$, sur laquelle $B$ est lipschitzienne. Désignant par $C$ la constante de Lipschitz de $B$ sur cette boule, on voit d'après (2.2) que

$$
<\frac{d U_{n}(t)}{d t}-\frac{d U_{m}(t)}{d t}, U_{n}(t)-U_{m}(t)>\leq C\left\|U_{n}(t)-U_{m}(t)\right\|^{2}
$$

Il en résulte aisément que $\left\|U_{n}(t)-U_{m}(t)\right\| \leq e^{C t}\left\|U_{n}(0)-U_{m}(0)\right\|$. En conséquence, un réel $T>0$ étant fixé, la convergence de la suite de fonctions $U_{n}($.$) vers U($.$) est$ uniforme sur $[0, T]$. Du caractère lipschitzien de $B$ sur $\mathcal{B}$ résulte alors la convergence uniforme de la suite $B\left(U_{n}().\right)$ vers $B(U()$.$) et de <B\left(U_{n}().\right), U_{n}()>$. vers $<B(U()),. U()>$. sur $[0, T]$. Le résultat annoncé découle alors d'un théorème élémentaire sur les suites de fonctions dérivables.

Remarque 2.3 Par hypothèse, on a $A^{*}=-A$, ce qui implique bien entendu $D\left(A^{*}\right)=D(A)$. Dans un souci de garder une certaine cohérence dans les notations, nous continuerons néanmoins à écrire $A^{*}$.

Théorème 2.1 On conserve notations et hypothèses de la Proposition 2.1 et l'on suppose que (2.9) est satisfait. On suppose de plus 
i. Il existe un opérateur $B_{0}: H \rightarrow H$ pour lequel toutes les solutions de l'équation

$$
\frac{d U}{d t}+A U+B_{0}(U)=0
$$

convergent fortement vers 0 .

ii. Pour toute suite $\left(U_{n}\right)_{n \in \mathbb{N}}$ d'éléments de $H$ convergeant faiblement vers un certain $U$ et vérifiant $\left\langle B\left(U_{n}\right), U_{n}>\underset{n \rightarrow+\infty}{\longrightarrow} 0\right.$, on a:

$$
\begin{gathered}
B\left(U_{n}\right) \underset{n \rightarrow+\infty}{\longrightarrow} 0 \text { faiblement dans } H, \\
B_{0}(U)=0 .
\end{gathered}
$$

Alors toutes les solutions $t \mapsto U(t)$ de l'équation (2.2) tendent vers 0 faiblement lorsque $t \rightarrow+\infty$.

Démonstration. On se donne $U_{0} \in H$ et l'on note $U(t)$ la solution de (2.2). D'après (2.7), la trajectoire $\mathcal{O}\left(U_{0}\right)=\{U(t), t \geq 0\}$ est bornée, donc faiblement compacte. Le Théorème 2.1 sera démontré dès lors que l'on aura prouvé que 0 est la seule valeur d'adhérence faible de $\mathcal{O}\left(U_{0}\right)$.

Soit donc $U_{1}$ une valeur d'adhérence faible de $\mathcal{O}\left(U_{0}\right)$ et $\left(t_{n}\right)$ une suite de limite $+\infty$ telle que $U\left(t_{n}\right) \longrightarrow U_{1}$ lorsque $t_{n} \rightarrow+\infty$. On fixe un réel $T>0$ et l'on considère sur $[0, T]$ la suite d'applications $\left(U_{n}\right)$ définie par $U_{n}(t)=U\left(t+t_{n}\right)$.

Proposition 2.2 Il existe une fonction $\bar{U}$ définie sur $[0, T]$ à valeurs dans $H$, continue lorsque $H$ est muni de la topologie faible $H_{w}$ et une sous-suite extraite de la suite $\left(U_{n}\right)$ (encore notée $\left(U_{n}\right)$ ) telle que

$$
U_{n} \underset{n \rightarrow+\infty}{\longrightarrow} \bar{U} \text { dans } C\left([0, T], H_{w}\right) .
$$

Démonstration. L'idée consiste à appliquer le théorème d'Ascoli. Puisque $\left\|U_{n}(t)\right\| \leq\|U(0)\|$ pour tout $t$ et tout $n$, la compacité pour la topologie faible de $H$ de l'ensemble $\left\{U_{n}(t), n \in \mathbb{N}\right\}$ est assurée pour chaque $t \in[0, T]$. D'autre part, cette inégalité montre que $\left(U_{n}\right)$ est une suite de fonctions à valeurs dans une boule de $H$. Or la topologie faible induite sur une partie bornée de $H$ est semi-métrisable pour la famille de semi-distances $\left(d_{W}\right)_{W \in H}$ définie par

$$
d_{W}(U, V)=\left|<U-V, W>_{H}\right| .
$$

Montrons l'équicontinuité de la suite $\left(U_{n}\right)$ pour cette famille de semi-distances. Soit $\varepsilon>0$ et $W \in H$. Il s'agit de trouver $\eta>0$ tel que pour tout $t, t^{\prime} \in[0, T]$ vérifiant $\left|t-t^{\prime}\right| \leq \eta$, on ait

$$
\forall n \in \mathbb{N},\left|<U_{n}(t)-U_{n}\left(t^{\prime}\right), W>\right| \leq \varepsilon
$$

D'après [1], proposition 2.2 p. 66 assurant l'identité entre solution d'une formulation 
variationnelle et solution mild, les $U_{n}$ satisfont la formule de variation des constantes

$$
U_{n}(t)=S_{0}(t)\left(U\left(t_{n}\right)\right)+\int_{0}^{t} S_{0}(t-s)\left(B\left(U_{n}(s)\right) \text { bigr }\right) d s
$$

où $\left(S_{0}(t)\right)_{t>0}$ est le semi-groupe engendré par $-A$. Pour tout $\left(t, t^{\prime}\right) \in[0, T]^{2}$ avec $t>t^{\prime}$ et $W \in H$ étant donné, on a donc

$$
\begin{gathered}
<U_{n}(t)-U_{n}\left(t^{\prime}\right), W>=<S_{0}(t)\left(U\left(t_{n}\right)\right)-S_{0}\left(t^{\prime}\right)\left(U\left(t_{n}\right)\right), W>+ \\
\int_{0}^{t^{\prime}}<\left(S_{0}(t-s)-S_{0}\left(t^{\prime}-s\right)\right)\left(B\left(U_{n}(s)\right)\right), W>d s+\int_{t^{\prime}}^{t}<S_{0}(t-s)\left(B\left(U_{n}(s)\right)\right), W>d s \\
=<U\left(t_{n}\right), S_{0}^{*}\left(t^{\prime}\right)\left[S_{0}^{*}\left(t-t^{\prime}\right)(W)-W\right]>+ \\
\int_{0}^{t^{\prime}}<B\left(U_{n}(s)\right), S_{0}^{*}\left(t^{\prime}-s\right)\left[S_{0}^{*}\left(t-t^{\prime}\right)(W)-W\right]>d s+\int_{t^{\prime}}^{t}<B\left(U_{n}(s)\right), S_{0}^{*}(t-s)(W)>d s,
\end{gathered}
$$

où $\left(S_{0}^{*}(t)\right)_{t>0}$ est le $C^{0}$ semi-groupe adjoint, engendré par $-A^{*}$. D'une part, $A^{*}$ étant anti-adjoint, on a $\left\|S_{0}^{*}(s)\right\| \leq 1$, d'autre part $S_{0}^{*}(\tau)(W)-W \underset{\tau \rightarrow 0}{\longrightarrow} 0$, si bien qu'il existe $\eta>0$ tel que $\left\|S_{0}^{*}(\tau)(W)-W\right\| \leq \varepsilon$ pour tout $\tau$ tel que $0 \leq \tau \leq \eta$.

Enfin, d'après (2.9) et (2.7), il existe une constante $C$ telle que $\left\|B\left(U_{n}(s)\right)\right\| \leq C$ pour tout $s$ et pour tout $n$. Il en résulte que dès que $\left|t-t^{\prime}\right| \leq \eta$ et pour tout $n \in N$, on a

$$
\left|<U_{n}(t)-U_{n}\left(t^{\prime}\right), W>\right| \leq\|U(0)\| \varepsilon+T C \varepsilon+\left(t-t^{\prime}\right) C\|W\| .
$$

Ce type d'inégalité prouve bien l'équicontinuité de la suite de fonctions $\left(U_{n}\right)$. La Proposition 2.2 résulte alors du théorème d'Ascoli (cf [7], p. 309).

Remarquons que l'on a aussi

$$
U_{n} \underset{n \rightarrow+\infty}{\longrightarrow} \bar{U} \text { dans } \mathcal{D}^{\prime}((0, T), H) .
$$

L'argument décisif dans la preuve du Théorème 2.1 est fourni ci-dessous.

Proposition 2.3 La fonction $\bar{U}$ ci-dessus satisfait la formulation variationnelle associée à l'équation

$$
\frac{d \bar{U}}{d t}+A \bar{U}=0
$$

vérifiant

$$
B_{0}(\bar{U}(t))=0 \quad \forall t \geq 0 .
$$

En particulier, $\|\bar{U}(t)\|$ est une constante.

Démonstration. Toujours d'après le caractère dissipatif de l'équation (2.2), la suite (précédemment extraite) de fonctions $\left(U_{n}\right)$ est bornée dans $L^{2}((0, T), H)$ puisque

$$
\int_{0}^{T}\left\|U_{n}(t)\right\|_{H}^{2} d t \leq T\left\|U_{0}\right\|_{H}^{2} .
$$


On peut donc en extraire une sous-suite convergeant faiblement dans $L^{2}((0, T), H)$ vers une certaine fonction $\bar{V}$. On a bien sûr

$$
U_{n} \underset{n \rightarrow+\infty}{\longrightarrow} \bar{V} \text { dans } \mathcal{D}^{\prime}((0, T), H)
$$

si bien que $\bar{V}=\bar{U}$ d'après (2.17).

En particulier,

$$
\forall t \in[0, T], U\left(t+t_{n}\right) \underset{n \rightarrow+\infty}{\rightarrow} \bar{V}(t) .
$$

L'inégalité d'énergie (2.8) donne

$$
\int_{0}^{T}<B\left(U\left(t+t_{n}\right)\right), U\left(t+t_{n}\right)>d t=\int_{t_{n}}^{t_{n}+T}<B(U(t)), U(t)>d t_{n \rightarrow t_{o+\infty}}^{\longrightarrow} 0 .
$$

A une sous-suite près, on en déduit que

$$
\text { p.p. } t \in[0, T],<B\left(U\left(t+t_{n}\right)\right), U\left(t+t_{n}\right)>\underset{n \rightarrow+\infty}{\longrightarrow} 0 .
$$

D'après l'hypothèse ii.(2.12), on en déduit que

$$
\forall W \in D\left(A^{*}\right), \text { p.p.t } \in[0, T],<B\left(U\left(t+t_{n}\right)\right), W>\underset{n \rightarrow+\infty}{\longrightarrow} 0 .
$$

Enfin, pour tout $W \in D\left(A^{*}\right)$ tout $t \in[0, T]$ et tout $n$, l'intégration de (2.10) sur l'intervalle $\left[t_{n}, t_{n}+t\right]$ donne

$$
\begin{gathered}
<U\left(t_{n}+t\right), W>-<U\left(t_{n}\right), W>+\int_{0}^{t}<U_{n}(s), A^{*} W>d s+ \\
\int_{0}^{t}<B\left(U\left(s+t_{n}\right)\right), W>d s=0 .
\end{gathered}
$$

Compte-tenu des convergences faibles de $\left(U_{n}\right)$ vers $\bar{V}$ dans $L^{2}((0, T), H)$ et de $\left(U\left(t_{n}\right)\right)$ vers $U_{1}$ dans $H$, des hypothèses (2.9) et (2.20) garantissant la convergence dominée dans la deuxième intégrale, le passage à la limite lorsque $n \rightarrow+\infty$ donne

$$
\begin{gathered}
\forall W \in D\left(A^{*}\right), \forall t \in[0, T], \\
<\bar{V}(t), W>-<U_{1}, W>+\int_{0}^{t}<\bar{V}(s), A^{*} W>d s=0 .
\end{gathered}
$$

Les deux faits suivants sont alors immédiats:

$$
\begin{gathered}
\forall W \in D\left(A^{*}\right), \lim _{t \rightarrow 0}<\bar{V}(t), W>=<U_{1}, W> \\
t \mapsto<\bar{V}(t), W>\in W^{1,2}((0, T), \mathbb{R}) \text { et } \frac{d}{d t}<\bar{V}(t), W>+<\bar{V}(t), A^{*} W>=0 .
\end{gathered}
$$

Ainsi $\bar{V}$, donc $\bar{U}$, satisfait (cf. [1]) la formulation variationnelle associée à l'équation

$$
\frac{d \bar{V}}{d t}+A \bar{V}=0
$$

ESAIM: Proc., Vol. 8, 2000, 137-150 
Puis d'après (2.18), (2.19) et l'hypothèse ii.(2.13), on en déduit $B_{0}(\bar{U}(t))=0$ pour tout $t \in[0, T]$, ce qui complète la preuve de la Proposition 2.2 .

Remarquons par ailleurs qu'étant donné un réel $T_{1}>T$, on construirait de même une fonction $t \mapsto \bar{V}_{1}(t)$ vérifiant (2.23) sur $\left[0, T_{1}\right]$ donc en particulier sur $[0, T]$. On en déduit, par unicité de la solution à un tel problème sur $[0, T]$, que $\bar{V}_{1}(t)=\bar{V}(t)$ sur $[0, T]$. On construit ainsi sans ambiguïté sur $[0,+\infty[$ une fonction $t \mapsto \bar{U}(t)$ satisfaisant la formulation variationnelle associée à l'équation $\frac{d \bar{U}}{d t}+A \bar{U}=0$ vérifiant $B_{0}(\bar{U}(t))=0$ pour tout $t \in[0,+\infty[$.

Le Théorème 2.1 en résulte, puisque l'on peut donc écrire

$$
\forall W \in D\left(A^{*}\right), \frac{d}{d t}<\bar{U}(t), W>+<\bar{U}(t), A^{*} W>+<B_{0}(\bar{U}(t)), W>=0
$$

si bien que $\bar{U}$ est une solution faible de l'équation

$$
\frac{d}{d t} U(t)+A U(t)+B_{0}(U(t))=0
$$

dont toutes les solutions sont censées tendre fortement vers $0:\|\bar{U}(t)\|_{t \rightarrow+\infty}^{\longrightarrow} 0$. Mais comme $\|\bar{U}(t)\|$ est une constante, on en déduit que $\bar{U}(t)=0$ pour tout $t \geq 0$. En particulier $U_{1}$, égal à $\bar{U}(0)$ d'après (2.14), est nul, ce qui achève la preuve du Théorème 2.2 .

\section{Applications à des systèmes hybrides}

\subsection{Pont roulant avec poutre.}

Dans cette première application, la méthode du problème factice va être employée à l'aide d'un opérateur linéaire, afin 'd'augmenter' la régularité de certaines solutions. Plus précisément, le problème étudié est situé dans un contexte monotone non linéaire, pour lequel l'application du principe de LaSalle (résolution d'un problème d'unicité par une méthode de multiplicateurs) requiert plus de régularité pour les solutions que celle fournie par la théorie standard des semi-groupes non linéaires, même pour une donnée initiale plus régulière. L'utilisation du problème factice, en choisissant un opérateur linéaire $B_{0}$, nous ramène à prouver la stabilité dans un cadre complètement linéaire, ce qui permet de disposer de solutions aussi régulières que l'on souhaite.

Le système étudié constitue une variante du modèle de pont roulant avec chariot, poutre et charge étudié dans [4], pour lequel on prend en considération un moment 
d'inertie. On obtient le système hybride suivant, constitué d'une équation d'EulerBernoulli couplée avec trois équations dynamiques aux extrémités:

$$
\left\{\begin{array}{l}
y_{t t}+y_{x x x x}-\left(a y_{x}\right)_{x}=0 \\
y_{x x}(1, t)+J y_{x t t}(1, t)=0 \\
M y_{t t}(1, t)-y_{x x x}(1, t)=-a(1) y_{x}(1, t) \\
y_{x}(0, t)=0 \\
m y_{t t}(0, t)+y_{x x x}(0, t)=-\alpha y(0, t)+f\left(y_{t}(0, t)\right)
\end{array}\right.
$$

où la force de tension $a$ est supposée de la forme $a(x)=M g+\rho(1-x)$.

Le but du problème est d'amener à l'équilibre l'ensemble poutre et charge à l'origine $y=0$ à partir d'une configuration arbitraire à l'instant $t=0$ à l'aide d'une loi de type feedback prenant en compte la position $y(0, t)$ et la vitesse $y_{t}(0, t)$ du chariot. On supposera que

$$
\alpha>0, f \text { est continue et décroissante sur } \mathbb{R} \text { et } s f(s)<0 \forall s \neq 0 \text {. }
$$

Suivant une procédure standard, le système (3.1) est converti en une équation d'évolution par rapport aux variables $\left(y, y_{t}, y_{t}(0, t), y_{t}(1, t), y_{x t}(1, t)\right)$. A cet effet, on considère l'espace

$$
H=\left\{U=(y, z, \eta, \xi, \theta) \in H^{2}(0,1) \times L^{2}(0,1) \times \mathbb{R}^{3} / y_{x}(0)=0\right\}
$$

muni de la norme hilbertienne

$$
\|U\|^{2}=\int_{0}^{1}\left(y_{x x}^{2}(x)+a(x) y_{x}^{2}(x)+z^{2}(x)\right) d x+\alpha y^{2}(0)+m \eta^{2}+M \xi^{2}+J \theta^{2},
$$

l'opérateur non borné $A$ défini par

$$
\begin{gathered}
D(A)=\left\{U=(y, z, \eta, \xi, \theta) \in H / y \in H^{4}(0,1), z \in H^{2}(0,1),\right. \\
\left.z_{x}(0)=0, \eta=z(0), \xi=z(1), \theta=z_{x}(1)\right\} \\
A(U)=\left(-z, y_{x x x x}-\left(a y_{x}\right)_{x}, \frac{1}{m}\left(y_{x x x}(0)+\alpha y(0)\right)\right. \\
\left.-\frac{1}{M}\left(y_{x x x}(1)-a y_{x}(1)\right), \frac{1}{J} y_{x x}(1)\right)
\end{gathered}
$$

puis l'opérateur $B$ défini sur $H$ par

$$
B(U)=\left(0,0,-\frac{1}{m} f(\eta), 0,0\right) .
$$

On vérifie aisément que toute solution $t \mapsto U(t)=(y(., t), z(., t), \eta(t), \xi(t), \theta(t))$ de 
l'équation d'évolution

$$
\left\{\begin{array}{l}
\frac{d U}{d t}+A U+B(U)=0 \\
U(0)=U_{0}=\left(y_{0}, z_{0}, \eta_{0}, \xi_{0}, \theta_{0}\right),
\end{array}\right.
$$

est telle que, au moins formellement, $y$ soit solution de $(3.1)$ avec $y(., 0)=y_{0}$, $y_{t}(., 0)=z_{0}$. On montre classiquement que l'opérateur $A+B$ de domaine $D(A)$ est maximal monotone sur $H$ et engendre donc un semi-groupe d'opérateurs contractants. En conséquence:

Proposition 3.1 Pour tout $U_{0} \in H$, l'équation (3.8) possède une solution faible unique $t \mapsto U(t)=(y(., t), z(., t), \eta(t), \xi(t), \theta(t))$. En outre, la fonction $E: t \mapsto$ $\frac{1}{2}\|U(t)\|^{2}$ est décroissante dérivable, avec

$$
E^{\prime}(t)=\eta(t) f(\eta(t)) .
$$

Lorsque $U_{0} \in D(A)$, on a $U \in W^{1, \infty}((0,+\infty), H) \cap L^{\infty}((0,+\infty), D(A)), \eta(t)=$ $y_{t}(0, t), \xi(t)=y_{t}(1, t)$ et $\theta(t)=y_{x t}(1, t)$.

Grâce à la méthode du problème factice, on prouve le

Théorème 3.1 Supposons (3.2) et que $J$, $\rho$ et $M$ sont assez petits. Alors pour tout $U_{0} \in H$, la solution correspondante $t \mapsto U(t)$ de (3.8) converge vers 0 fortement dans $H$ lorsque $t \mapsto+\infty$.

Remarque 3.5 Comme il a été mentionné ci-dessus, une idée naturelle consisterait à appliquer le principe de LaSalle, au moins lorsque $U_{0} \in D(A)$, ce qui conduirait à montrer que $y=0$ est la seule solution du système

$$
\left\{\begin{array}{l}
y_{t t}+y_{x x x x}-\left(a y_{x}\right)_{x}=0 \\
y_{x x}(1, t)+J y_{x t t}(1, t)=0 \\
M y_{t t}(1, t)-y_{x x x}(1, t)=-a(1) y_{x}(1, t) \\
y_{x}(0, t)=0 \\
y_{t}(0, t)=0 \\
y_{x x x}(0, t)=-\alpha y(0, t)
\end{array}\right.
$$

qui correspond aux solutions de (3.1) d'énergie constante. Comme il sera précisé cidessous, il existe un multiplicateur permettant de résoudre au moins formellement ce problème, mais il nécessite que $y \in H^{5}(0,1)$, qui est une régularité non garantie par la Proposition 3.1, même en prenant une donnée initiale plus régulière, étant dans un contexte non linéaire, contrairement au cas linéaire où la solution "hérite" de la régularité de la donnée initiale ( $c f$. [2]). C'est essentiellement cette propriété qui sera exploitée pour prouver la stabilité pour le problème factice.

Remarque 3.6 L'injection $D(A) \rightarrow H$ est clairement compacte. Il en résulte que les orbites des solutions fortes de (3.8) sont précompactes pour la topologie de $H$. Par un raisonnement classique, le Théorème 3.1 résultera de la convergence faible 
des solutions fortes et d'un argument de densité et contraction.

Démonstration du Théorème 3.1. (2.9) et (2.12) se prouvent de façon élémentaire.

On se donne ensuite un réel $k>0$ et l'on considère l'opérateur linéaire $B_{0}$ défini sur $H$ par

$$
B_{0}(U)=\left(0,0,-\frac{1}{m} k \eta, 0,0\right)
$$

Il est clair que (2.13) est réalisé, si bien que d'après le Théorème 2.1 et la Remarque 3.6, le Théorème 3.1 sera démontré dès lors que l'on aura prouvé que toutes les solutions $t \mapsto U(t)$ de l'équation

$$
\left\{\begin{array}{l}
\frac{d U}{d t}+A U+B_{0}(U)=0 \\
U(0)=U_{0}
\end{array}\right.
$$

convergent fortement vers 0 lorsque $t \rightarrow+\infty$. A cet effet, on montre aisément que l'opérateur $B_{0}$ est maximal monotone sur $H$. Donc l'opérateur $A_{0}: D\left(A_{0}\right) \rightarrow H$ défini par

$$
\left\{\begin{array}{l}
D\left(A_{0}\right)=D(A) \\
A_{0} U=A U+B_{0} U
\end{array}\right.
$$

est maximal monotone sur $H$. Pour des raisons évidentes de densité et de contraction, il suffit d'établir la convergence forte des solutions de (3.11) pour tout $U_{0} \in D\left(A_{0}^{2}\right)$.

Fixons $U_{0} \in D\left(A_{0}^{2}\right)$, introduisons l'espace de Hilbert $H_{1}=\left(D\left(A_{0}\right),\|\|_{A_{0}}\right)$ et l'opérateur $A_{1}: D\left(A_{1}\right) \subset H_{1} \rightarrow H_{1}$ défini par

$$
\left\{\begin{array}{l}
D\left(A_{1}\right)=D\left(A_{0}^{2}\right) \\
A_{1} U=A_{0} U
\end{array}\right.
$$

D'après [2], théorème VII.5, on sait que $A_{1}$ est un opérateur maximal monotone sur $H_{1}$. L'équation

$$
\left\{\begin{array}{l}
\frac{d V}{d t}+A_{1} V=0 \\
V(0)=V_{0}
\end{array}\right.
$$

possède donc une unique solution pour tout $V_{0} \in H_{1}$, et pour $V_{0}=U_{0}$, celle-ci n'est autre que la solution $t \mapsto U(t)$ de (3.11).

D'autre part, l'injection $D\left(A_{1}\right) \rightarrow H_{1}$ est clairement compacte. L'ensemble $\omega$-limite $\omega_{1}\left(U_{0}\right)$ (pour la topologie de $H_{1}$ ) de l'orbite $\{V(t), t \geq 0\}=\{U(t), t \geq 0\}$ est donc non vide, inclus dans $D\left(A_{1}\right)$ par fermeture forte-faible du graphe de $A_{1}$, invariant sous l'action du semi-groupe engendré par $A_{1}$ et d'après le principe de LaSalle 
appliqué à la fonction de Liapounov $t \mapsto \frac{1}{2}\|V(t)\|_{H_{1}}^{2}$, pour tout $W_{0} \in \omega_{1}\left(U_{0}\right)$, la solution $t \mapsto W(t)=(y(., t), z(., t), \eta(t), \xi(t), \theta(t))$ de (3.12) vérifiant $W(0)=W_{0}$ est telle que $\frac{1}{2}\|W(t)\|_{H_{1}}^{2}$ soit constant.

Comme on vérifie sans peine que $<A_{0} W, W>_{H}=\frac{k}{m} \eta^{2}$, on en déduit que $y_{t}(0, t)=$ 0 , donc $t \mapsto y(t)$ satisfait (3.10). Puisque dans ce cadre on a $W(t) \in D\left(A_{1}\right)$ pour tout $t \geq 0$, on peut dériver en espace la première équation de (3.10) et en utilisant le multiplicateur $(x-M-1) y_{x x}$, des inégalités intégrales conduisent, lorsque $J, \rho$ et $M$ sont assez petits, à $y \equiv 0$ (pour les détails, voir [6]). On en déduit $\omega_{1}\left(U_{0}\right)=\{0\}$. Ainsi, $V(t) \rightarrow 0$ lorsque $t \rightarrow+\infty$ pour la topologie de $H_{1}$, donc pour la topologie de $H$, ce qu'il fallait démontrer.

\subsection{Pont roulant avec câble.}

Dans cette deuxième application, le principe du problème factice est (notamment) utilisé pour étudier la convergence des solutions faibles dans un cadre non contractant. On considère le modèle de pont roulant avec câble développé dans [3]. Il s'agit du système hybride:

$$
\left\{\begin{array}{l}
y_{t t}(x, t)-\left(a y_{x}\right)_{x}(x, t)=0, t>0,0<x<1 \\
\left(a y_{x}\right)(1, t)+M y_{t t}(1, t)=0 \\
\left(a y_{x}\right)(0, t)-m y_{t t}(0, t)=f\left(y_{t}(0, t)\right)-\alpha y(0, t) .
\end{array}\right.
$$

Dans [3], il a été prouvé que lorsque $f$ est continue sur $\mathbb{R}, s f(s) \geq 0$ pour tout réel $s$ et que le taux d'accroissement de $f$ est majoré sur tout compact, alors (3.13) est bien posé. De plus, si $f$ est localement croissante au voisinage de 0 avec $s f(s)>0$ sur un intervalle $] 0, \delta]$, alors toutes les solutions fortes de (3.13) tendent fortement vers 0 .

Rappelons que la question de la stabilité des solutions faibles demeurait ouverte dans la mesure où l'absence de contraction ne permettait pas d'utiliser un raisonnement basé sur la densité et la méthode employée pour prouver la stabilité des solutions fortes nécessitait a priori une certaine régularité que ne possèdent pas les solutions faibles.

On va alors introduire un opérateur factice $B_{0}$ monotone auquel on pourra associer un semi-groupe contractant, rendant exploitable cette propriété de densité.

On conserve les hypothèses mentionnées ci-dessus portant sur $f$, assurant l'existence des solutions. On convient (pour alléger l'écriture) que la masse $m$ du chariot vaut 1 .

Théorème 3.2 Supposons que

$$
\forall s>0, f(s)>0 \text {. }
$$

i. Toutes les solutions fortes et faibles de 3.13 convergent vers 0 faiblement dans $H$ lorsque $t \rightarrow+\infty$. 
ii. Supposons de plus que

$$
f \text { est croissante (largement) sur un voisinage de } 0 .
$$

Alors toutes les solutions faibles de 3.13 convergent vers 0 fortement dans $H$ lorsque $t \rightarrow+\infty$.

Remarque 3.4 Ce théorème est, sur certains aspects, moins fort que le théorème de stabilité pour les solutions fortes établi dans [3] dans la mesure où (3.14) n'était pas exigé. En revanche, aucune hypothèse de monotonie n'est nécessaire. Par ailleurs, il concerne également les solutions faibles.

Démonstration. Vérifions les hypothèses du Théorème 3.1. Tout d'abord, si $U=$ $(y, z, \eta, \xi) \in H$ est tel que $\|U\|^{2} \leq C$, alors $\eta^{2} \leq C$. La propriété (2.9) en résulte, du fait que $f$, continue sur $\mathbb{R}$, transforme bornés en bornés.

Considérons ensuite l'application $f_{0}$ définie sur $\mathbb{R}$ par $f_{0}(s)=s$ pour $s>0$ et $f_{0}(s)=0$ pour $s \leq 0$ et l'opérateur $B_{0}$ défini sur $H$ par

$$
B_{0}(U)=\left(0,0, f_{0}(\eta), 0\right) .
$$

Si $U_{n}=\left(y_{n}, z_{n}, \eta_{n}, \xi_{n}\right)$ est une suite convergeant faiblement vers $U=(y, z, \eta, \xi)$ dans $H$ et telle que $<B\left(U_{n}\right), U_{n}>\rightarrow 0$ lorsque $n \rightarrow+\infty$, alors, en particulier, $\eta_{n} \rightarrow \eta$ et $f\left(\eta_{n}\right) \eta_{n} \rightarrow 0$. Puisque $f$ est continue sur $\mathbb{R}$, on a $f\left(\eta_{n}\right) \rightarrow f(\eta)$ et il en résulte que $f(\eta) \eta=0$. Donc ou bien $\eta=0$, ou bien $f(\eta)=0$. Dans les deux cas, on a $f(\eta)=0$ et on a alors évidemment $f\left(\eta_{n}\right) \rightarrow 0$ et (3.15) en résulte.

Enfin, puisque $f(\eta)=0$, c'est que, d'après (3.27), $\eta \leq 0$. Donc $f_{0}(\eta)=0$ et $B_{0}(U)=0$. Donc (2.13) est satisfait.

Quant à l'hypothèse i., elle est réalisée puisque $f_{0}$ vérifie les hypothèses rappelées ci-dessus assurant la stabilité forte des solutions fortes d'après le résultat de stabilité obtenu dans [3]. L'application $f_{0}$ étant croissante sur $\mathbb{R}$, il en résulte que le semigroupe engendré par $A+B_{0}$ est contractant sur $H$ et le résultat de stabilité forte s'étend aux solutions faibles.

Ainsi, les hypothèses du Théorème 3.1 sont satisfaites et on en déduit que toutes les solutions de (3.13) tendent faiblement vers 0 .

Supposons enfin (3.15). Pour une solution faible

$$
t \mapsto U(t)=(y(., t), z(., t), \eta(t), \xi(t)),
$$

la convergence faible vers 0 a bien entendu pour conséquence $\eta(t) \underset{t \rightarrow+\infty}{\longrightarrow} 0$. Donc à partir d'un certain instant $t_{0}$, la troisième composante $\eta(t)$, sur laquelle agit exclusivement l'opérateur $B$, appartient à un intervalle sur lequel $f$ est croissante. On procède alors comme dans [3] pour se ramener à un semi-groupe contractant dont toutes les solutions fortes tendent vers 0. D'où ii., par un argument de densité et 
contraction.

Remerciements. L'auteur tient particulièrement à remercier les Professeurs F. Conrad et A. Haraux pour de très fructueuses conversations et suggestions.

\section{Bibliographie}

[1] Bensoussan A. et al, Representation and Control of Infinite Dimensional Systems, Vol. I, Birkhäuser, (1992).

[2] Brézis H., Analyse Fonctionnelle, Théorie et Applications, Masson (1983).

[3] Feireisl E. et O'Dowd G., Stabilization of a Hybrid System with a Nonlinear Nonmonotone Feedback, ESAIM: COCV, Vol. 4 (1999).

[4] Feireisl E. et O'Dowd G., Stabilization of a Hybrid System: an Overhead Crane with Beam Model, Portugaliæ Mathematica, à paraître.

[5] Haraux A., Semi-linear Hyperbolic Problems in Bounded Domains, Mathematical reports Vol 3, Part I. J. Dieudonné Editor, Harwood Academic Publishers, Gordon \& Breach (1987).

[6] O'Dowd G., Stabilisation de systèmes distribués au moyen de contrôles dissipatifs non monotones, Thèse de l'Université de Nancy I (1999).

[7] Schwartz L., Analyse, Topologie générale et analyse fonctionnelle, Collection Enseignement des Sciences, 11, Hermann, (1970).

[8] Slemrod M.,Weak Stabilization Decay via a "Relaxed Invariance Principle" for a Wave Equation with Nonlinear Nonmonotone Damping, Proc. Roy. Soc. Edinburgh 113A, pp. 87-97 (1989).

[9] Vancostenoble J., Weak asymptotic stability of second order evolution equations by nonlinear and nonmonotone feedbacks, SIAM J. Math. Anal. Vol. 30, No. 1, pp. 140-154 (1999). 